\title{
Application of Green Design in Interior Design
}

\author{
Shanshuang Li \\ School of Art and Design \\ Huanghe Science and Technology College \\ Zhengzhou, China 450046 \\ E-mail: 479774980@qq.com
}

\begin{abstract}
Green design is an advanced design idea, which is developed with the concept of sustainable development. It emphasizes the protection of natural ecology, the full use of resources, people orientation and environment protection. With haze and fog shrouding us, the green has become a common concern of the world. The green design is becoming a new way of design and surging forward. With green design as research object, the paper has comprehensively discussed the application of green design in interior design from many aspects, including overview of interior design, requirements of scientific humanity and application of "subtraction design" and etc.
\end{abstract}

\section{Keywords—green design; interior design; application}

\section{INTRODUCTION}

\section{A. The Historical Development of Interior Design}

Interior design is a new and hot industry in recent years. At present, the situation of centering on economic construction has provides an unprecedented space for the development of interior design. As an independent discipline, interior design has developed rapidly. But it has existed that people intentionally arrange their room for living and production activities, decorate it and make it into desired environment since the beginning of human civilization.

In our country, from the early primitive society to the feudal society, after thousands of years of continuous development and changes of Chinese ancient civilization, interior design has gradually formed its unique style characteristics in China. With the feature of adjusting measures based on local conditions and applying for different purposes according to types, our interior design has different geographical and cultural characteristics, rich, various, simple and natural. For example, Beijing's Courtyard, Sichuan's Mountain House, Dai people's Ganlan House and Shanghai's Linong Housing reflect an elegant interior space mood and atmosphere. There are many valuable achievements for reference in architectural decoration design and production and etc.

In foreign countries, in the early fifteenth century, interior decoration adopted the decoration style of Ancient Greece, Ancient Rome, the Oriental and Gothic. It closely integrates architecture, carving and painting with new techniques of expression, creating an elegant and gorgeous interior decoration effect. In the middle of the 17th century, it gradually evolved into "Baroque" style. Based on the spirit of romanticism, interior decoration shows luxury and magnificent characteristics. At the beginning of the 18th century, interior decoration tended to be kind, ingenious, with much curve modeling, delicate carvings, simple, elegant and mild color and gorgeous decoration. In the late 18th century, decoration concepts have changed with the arrival of the industrial revolution. People began to question the luxury decoration, traditional values and aesthetic concepts and to pursue simple, concise and amiable interior decoration, and they advocated that interior decoration could not be separated from building noumenon.

In 1919 Germany's Bauhaus School discarded traditional concepts and advocated to stress functions, which promote the application of modern technologies and new materials. Bauhaus School put forward new concepts in architecture and interior design to adapt to the industrial society. In 1930s, Corbusier advocated "Mechanical Aesthetics" (also known as Functionalism or International Style), and thought modern design shouldn't contain any elements belonging to "traditional decoration" and emphasized that "Mechanical Aesthetics" is both a spirit and an inspiration of style.

\section{B. Connotation of Interior Design}

Interior design is to create a comfortable and beautiful indoor environment that can meet people's demand for material and spiritual life with materials, technical means and architectural aesthetics principles according to building environment, using properties and corresponding standards. It continues and deepens the architectural design and recreates room space and environment. Specifically it is:

- To create a reasonable internal space relationship with reasonable layout, convenient flow line and clear gradation.

- To create a comfortable interior environment that meet people's needs for environmental temperature, ventilation, soft light and sound and etc.

- To meet people's spiritual needs for environment mood, artistic conception and culture.

Therefore, the interior design is not just a simple processing of wall, roof, ground and other interface forms. Its essence is to build an ideal space.1 Interior design is sourced and separately from architectural design. Its creation 
is always conditioned by building, so it is a freedom in "cage".

In simple terms, interior design mainly includes space design, decoration design, display design and physical environment design. The physical environment design involves in indoor climate, heating, ventilation, temperature and humidity adjustment and etc.

Interior design has a very close relationship with architectural design, and they are deeply interpenetrated with one another. They are consistent with each other in design idea, service aim and evaluation criteria. Usually architectural design is the premise of interior design. Interior design, as a relatively independent emerging discipline, has the following characteristics:

- Directly and closely influence people's mind and body;

- Thoroughly consider the constitution factors of the indoor environment;

- Detailedly and profoundly reflect the aesthetic elements of the design.

- To be more prominent in the change of interior function, material and equipment than that of architectural design, to be with short update cycle, fast rhythm and need to introduce new concepts, such as "dynamic design" and "latent design".

- To be with high technology content and cultural content; moderate cultural content is the charm and the aura of a piece of work. The cultural content brings people psychological enjoyment and spiritual pleasure. The technical content brings people physical enjoyment and use convenience. At the same time, design is also closely coordinated with auxiliary facilities. They complement each other and are integrated into one.

\section{The Development Trend of Interior Design}

The development of art is endless. The interior design, as an applied art, continuously absorbs new art flavors, follows the development of times and social progress, and closely watches people's material needs and spiritual needs. Its development trend mainly includes the following aspects:

1) High-tech intelligent design trend. With the development of society and the progress of science and technology, it will be very convenient and frequent to use modern intelligent information equipment, which is an irresistible historical trend. High tech has greatly enriched the expressive force and performance of indoor environment. People can take advantage of high technologies to combine humanities, art, nature and form elements and apply to people's living environment, and create a new form of art and ecological environment. For example, intelligent office and intelligent residence will gradually develop into the general direction of interior design.

2) The design trend with more emphasis on cultural connotation. The more highly developed the post-industrial and information society is, the more urgently people need culture. Therefore, the interior design should blend the spirit of the times and historical context together, forging an interior environment with a sense of times, local style and national characteristics. Use modern design concepts to reinterpret and pass down interior design and make it filled with cultural connotation, so as to improve it into a new level in style and taste and promote the forming of new design style.

3) The design trend of sustainable development and ecological ideas. Ecological, environmental and sustainable development has become the most urgent research topic faced by interior designers in twenty-first Century. It is a real problem of global concern that how can we protect our environment, maintain the balance of ecosystem, and use resources reasonably and effectively. It is also the responsibility of modern designers. The concept of ecology will occupy more and more important position in the design of the future, and will gradually become the mainstream of interior design.

4) The design trend of owner and public participation. Professional design will be further deepened and standardized, meanwhile the tendency of owner and public participation will be strengthened. It is because the creation of indoor space environment can not be separated from user's needs for life and production activities. Designers should hear the ideas and requirements of the user, so that they can reach a consensus in design concept and the design can meet the needs of owners. As a result, such design works are more effective and perfect in use function.

\section{How to REALIZE GREEN DESIGN IN INTERIOR DESIGN}

\section{A. Scientific and People-Oriented Design Requirements}

Interior design is a product of modern science and technology and culture, which should fully reflect the value of people. Make design based on the people-oriented concept study people's physiological and psychological characteristics, seek environment structure fitting for users and create a comfortable, safe, practical, environmental protection interior environment.

1) Selecting green materials. Material is the skeleton of the interior design and the bridge for successful design. If designers select and apply materials rightly, they are able to perform wonders and make perfect design.

All kinds of materials in life can be used in interior decoration, such as stone, wood and composite materials. Stone and wood have been used since the primitive society, while composite materials are modern elements. However, not all materials can be used for a good environment. Green materials are safe, environmentally friendly, and coordinated with environment, so we can use green materials to create a safe, comfortable, pleasant and healthy indoor environment.

Green Material (GM) is also known as Environmental Conscious Material (ECM) and Eco-material. This kind of material has good usability and functions, less pollution to 
ecology and environment, and can reduce consumption of resources and energy. They are beneficial to human health, and have high recycling rate and can be degradable for recycle. In the whole process of preparation, use, abandon and recycle, they are coordinated and coexisted with environment. We should not only develop high-tech materials with direct functions of purifying environment and repair but also transform traditional materials of large consumption and coverage in order to make them "environmental".

Green Material is a relative concept, which is still in the process of continuous development and improvement. In general, green material should have three obvious characteristics: a good usability, high resource utilization rate and no side effect to ecological environment. Therefore, it is an important content in the research of green materials to emphasize the compatibility between material and environment and to develop environment friendly new materials.

In addition, the application of natural materials is an important method in green interior design. Modern interior design particularly lays emphasis on the application of texture of natural materials. Designers can highlight the texture of natural materials with the selection of surface materials and material processing, boldly express the texture of wood, metal and other materials and deliberately display material texture and true color. They may be original and rough, or crafted, or ardent and simple. They affect people's emotions and compensate people living in city but longing for hometown and nature.

2) Creating scientific and people-oriented space layout, ensuring convenience and comfort, and maintaining the privacy of family members. The range of activity, also called action domain, for people's work and life is an important factor in determining the size of indoor space. The action domain of human body measured by various methods is the basic data for study of ergonomics.

In the selection of detail size for action domain, designers should consider the security of people's movements and activities and the appropriate size for the majority of people under different space and enclosure, and stress the premise of safety. Designers shall take advantage of the ratio scale of ergonomics to design people-oriented functions. In any environment designers shall compare and analyze the ratio scale of environmental space with this scale, which shall become a professional habit of our designers. "People orientation" is always the theme. With the functional requirements of people, designers can use the plane space and three-dimensional space as far as possible, follow the principle of building structure, and position the functions of space that does not affect the permissible scope of the housing structure, so that the whole design is convenient, comfortable and practical.

3) Making scientific layout and careful allocation of indoor lighting. Light is the source of life. Light is a material with intelligence. Light is the element of the living environment. From human's understanding of the light to the use of light and creating a special light environment, we can see the light plays an indispensable role in the interior design.

It is easy to cause light pollution and energy loss if the design for indoor light environment is improper. Designers shall arrange different lighting equipment according to functions in lighting system.

The principles of interior lighting design: there are three kinds of lighting in domestic decoration, including centralized light source, auxiliary light source and illuminating-all-things light source. Not a single one can be omitted. Designers shall use them in a cross and combined way, and the proportion of their brightness is about 5 to 3 to 1.

Centralized light source: the light of centralized light source is sourced from direct illuminating lamp. The lamp lights certain limited area with direct centralized light, so that people can see things and motions clear, especially when they are working, reading or eating. This kind of lamps includes spotlight, track lights, and etc.

Auxiliary light source: the illumination of centralized light source is high in brightness, so eyes are easy to get tired in this environment for a long time. At this moment, auxiliary light source is very useful. For example, use floor lamp and book light to reconcile indoor light difference and make eyes feel comfortable. Generally speaking, the light of scattered light can be used together with the light of direct light.

Illuminating-all-things light source: the ceiling lamp is an illuminating-all-things light source. Usually it is the main light in room, and it is also known as background light. It can improve the indoor light to certain brightness, and make the entire room with same light, so that it will not produce a clear shadow. There is no series contrast between lighting area and non-lighting area. However, it shall be used together with other lights, because it is not very bright generally. Its lightness is relative low compared with other light sources.

Grasp the performances and use effects of all kinds of lamps and lanterns, and at same time avoid misuse, because the blind pursuit of effect and the atmosphere, especially in use of color light sources, may make eyes dazzling and cause a effect neither fish nor fowl. Therefore, designers should study the lighting system of interior environment, so as to make scientific layout and elaborate arrangement.

Nowadays, interior design should be people-oriented, with materials and light lighting as spiritual purpose. Create space that are suitable for dimension of human body and close to modern people's psychology as far as possible, use natural lighting, natural energy, green materials, and pay attention to indoor greening and reasonably use underground space. Create a spatial environment of sustainable development and guarantee the coordinated development of nature and people. 


\section{B. Application of "Subtraction Design"}

Subtraction design is to replace irregular indoor edge and corner structures, even to pull down insignificant nonbearing wall, and use geometric forms to unify and generalize the interior environment, and make it even, tidy and pleasing. It breaks the tradition and pays more attention to function design, abandons false decoration, advocates concise decoration and reduces decoration elements to a minimum. Compared with traditional design, it is no longer to fill various decorative materials in indoor space, but to use simple images and eliminate unnecessary decoration and focus on careful scrutiny of the proportion of various indoor components. In addition, it emphasizes the form beauty of indoor space environment and the original beauty of materials and the contrast effect in height, size and direction of each unit and the empty and full effect, showing a sense of freedom, freshness and cleanness.

Interior design emphasizes subtraction design. But it does not mean to dispense with all complicated decorations. It is a creative process of digestion and absorption, so designers shall read a lot and conceive repeatedly. For the sake of space vision, make a lighting effect of large scope beyond the original limit. The key to "subtraction design" is to highly generalize and define spatial form to the hilt, to reduce uncorrelated factors and avoid interference. Only by removing non-essentials and presentation, can designers abstractly generalize, sum up, simplify and exaggerate real environment and grasp the most individual and essential characteristics. "Subtraction design" can create a concise, fresh, simple and open space atmosphere with circulating air environment and making people feel relaxed and comfortable.

\section{REFERENCES}

[1] Pan Tianshou: "Aesthetics of Color ", Beijing: People's Fine Arts Publishing House, ed.1998.

[2] Chang Huaisheng, Environmental Psychology and Interior Design, Beijing: China Building Industry Press, 2000 